\title{
6. UNDERWAY GEOPHYSICAL MEASUREMENTS FROM GLOMAR CHALLENGER, DEEP SEA DRILLING PROJECT LEG 50, AND MULTICHANNEL SEISMIC-REFLECTION PROFILE OF R/V METEOR, CRUISE 3902
}

\author{
Edward L. Winterer, Scripps Institution of Oceanography, La Jolla, California \\ Yves Lancelot, Département de Géologie Dynamique, Université Pierre et Marie Curie, Paris, France, and \\ Scripps Institution of Oceanography, La Jolla, California \\ and \\ Karl Hinz, Bundesanstalt für Geowissenschaften und Rohstoffe, Hannover, Federal Republic of Germany
}

We present here the navigation and underway geophysical measurements (bathymetry, magnetics, seismic-reflection profiles) obtained from Glomar Challenger during Leg 50, and a multichannel seismic-reflection profile obtained by R/V Meteor on cruise 3902 along a track close to a line between Sites 415 and 416 .

The Glomar Challenger left Funchal, Madeira, at 2335 hours on 11 September 1976 and sailed to the area of Site 415 , where a short site survey was made; the acoustic beacon was dropped at Site 415 at 1346 hours on 13 September. The ship left the site at 2305 hours on 28 September and steamed to Site 416 , along a track about $50 \mathrm{~km}$ west of the multifold seismic line run by Meteor. The seismic-profile records along this part of the Glomar Challenger track show for the most part only the relatively shallow reflectors, owing to imperfect functioning of the equipment. The ship then turned east from this track to a position close to Site 370 (drilled on Leg 41), where annchal to make a crew change at 2155 hours on 26 October, arriving at 1600 hours on 28 October. The ship departed Funchal again at 0025 hours on 29 October and arrived at the site at 1459 hours on 30 October. Drilling operations ended at Site 416 at 1500 hours; the ship steamed across Site 416 while profiling, then on to Funchal to end Leg 50 on 10 November. The ship's track is shown in Figure 1, and navigation data are shown in Table 1. Errors are generally less than 1 mile. Table 1 also gives the regional magnetic values at each navigational point, using the reference field of $\mathrm{Fa}$ biano and Peddie (1969).

Bathymetric soundings, magnetic measurements, and seismic-profile records were made over the entire track, except for the immediate vicinity of the port of Funchal. Th magnetic and bathymetric profiles are displayed together in Figure 2, and the seismic-profiler records are shown in Figure 3 in the back pocket of this volume. The seismic records were recorded on a dry-paper EDO recorder, using two air guns (20 and 40 in $^{3}$ firing chambers) as a sound source. Depths are labeled along the sides of the records in two-way reflection time, in seconds. Times and dates are shown along the records, and the drill sites and certain major bathymetric features are labeled. The times during which the seismic profiler was recording are shown beneath the bathymetric profile (Figure 2) by black bars.

The R/V Meteor multifold profile is shown in Figure 4 , and a track chart for the profile is shown in Figure 5.

The technical specifications for the Meteor 3902 profile are:

Sound source: Two 300 -in ${ }^{3}$ air guns

Streamer: 12-trace Geomechanique, 1500 meters long, 1200 hydrophones

Shot-point spacing:

Recording:

Processing:

\section{0 meters}

24-trace MDS-8 digital unit

Raytheon RDS 500 computer

Input tape format SEGB9, 800BPI, sampling rate 4 milliseconds

Steps:

(1) Uniprep, (2) Suprsor, (3) Amplitude correction, (4) Velocity analysis, (5) Horizontal stacking, 12-fold, (6) Frequency filter: low cut $8-15 \mathrm{~Hz}$, high cut $55-65 \mathrm{~Hz}$, (7) Display

Navigation: Integrated Indas satellite navigation system.

In order that the reader may be free to interpret the profiler record without any guidance from us, none of the key reflectors has been labeled. A discussion of the correlation between drill results and seismic reflectors is included in the site reports in this volume. An interpretation of the Meteor 3902 profile by the shipboard party is shown in a figure in the Site 416 report.

\section{ACKNOWLEDGMENTS}

The efforts of the DSDP laboratory technicians, under the direction of Gerald Bode, are greatly appreciated.

\section{REFERENCES}

Fabiano, E. G., and Peddie, N. U., 1969. Grid values of total magnetic intensity I.G.R.F., 1965: U.S. ESSA Tech. Rept., v. 38 , p. 55 . 


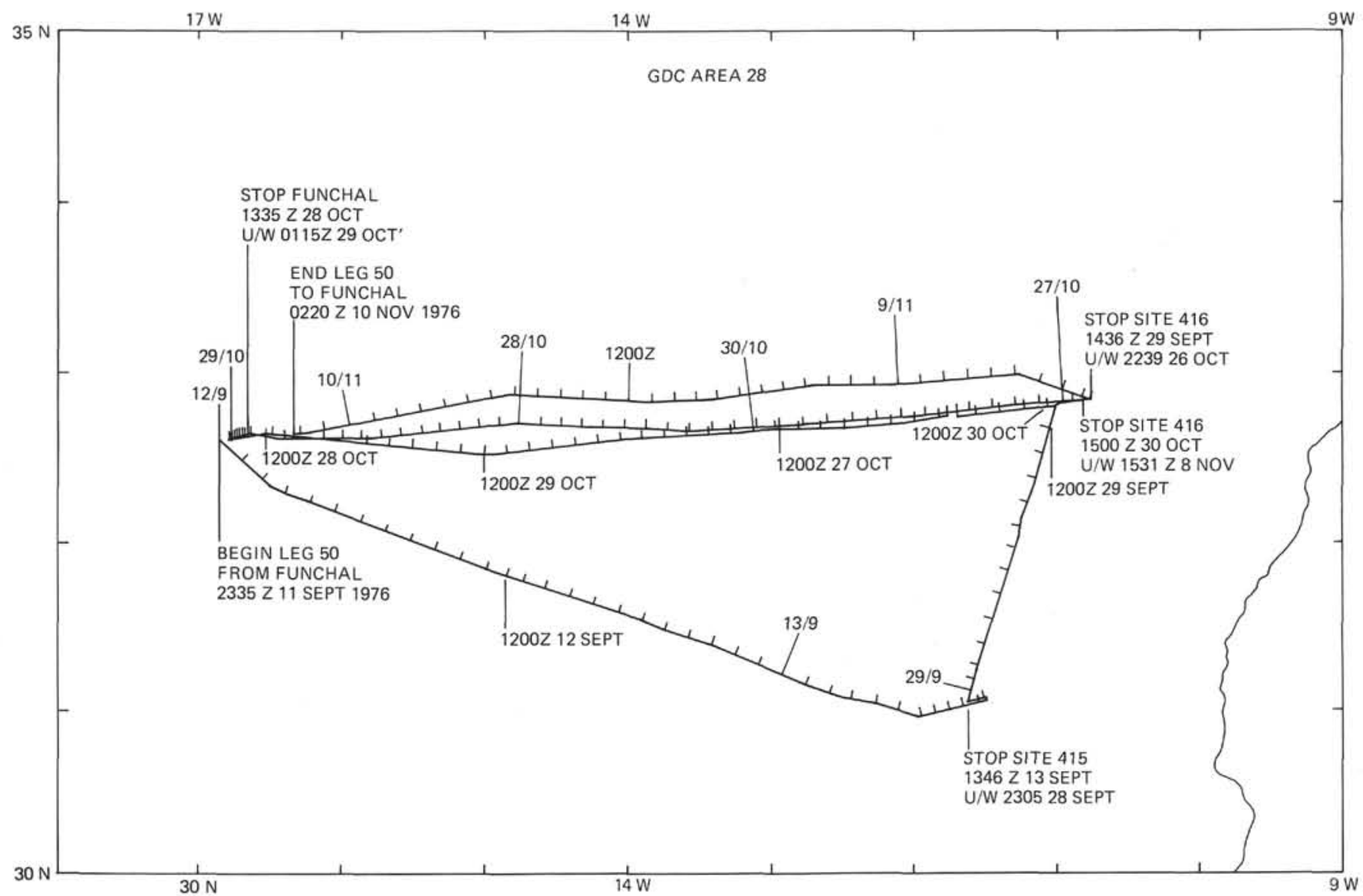

Figure 1. Track of Glomar Challenger during Leg 50.

TABLE 1

Navigation Data, D/V Glomar Challenger, Leg 50

\begin{tabular}{|c|c|c|c|c|c|c|c|c|c|c|c|c|c|c|c|c|}
\hline \multirow[b]{2}{*}{ Date } & \multirow[b]{2}{*}{ Time } & \multicolumn{2}{|c|}{ Latitude } & \multicolumn{2}{|c|}{ Longitude } & \multirow[b]{2}{*}{ Dist. } & \multicolumn{2}{|c|}{ Actual } & \multicolumn{2}{|c|}{ Drift } & \multicolumn{2}{|c|}{$\mathrm{Dr}$} & \multirow[b]{2}{*}{ Cmnt. } & \multicolumn{3}{|c|}{ Drift } \\
\hline & & Deg. & Min. & Deg. & Min. & & Speed & Cse. & Speed & Hed. & Speed & Cse. & & Dist. & Time & No \\
\hline 11 Sept. 76 & 2335 & 32 & 38.25 & -16 & 54.39 & 0.0 & 5.8 & 137 & 1.4 & 186 & 5.0 & 125 & Satl & 0.0 & 0.0 & 3 \\
\hline 11 Sept. 76 & 2344 & 32 & 37.6 & -16 & 53.7 & 0.9 & 9.7 & 132 & 1.4 & 186 & 9. & 125 & $\mathrm{C} / \mathrm{S}$ & & & 4 \\
\hline 12 Sept. 76 & 219 & 32 & 20.8 & -16 & 31.6 & 26.0 & 9.4 & 118 & 1.4 & 186 & 9.0 & 110 & $\mathrm{C} / \mathrm{C}$ & & & $J$ \\
\hline 12 Sept. 76 & 256 & 32 & 18.04 & -16 & 25.51 & 31.8 & 9.8 & 109 & 0.8 & 95 & 9.0 & 110 & Satl & 4.6 & 3.3 & 7 \\
\hline 12 Sept. 76 & 442 & 32 & 12.49 & -16 & 6.22 & 49.0 & 9.5 & 113 & 0.6 & 150 & 9.0 & 110 & Satl & 1.4 & 1.8 & 9 \\
\hline 12 Sept. 76 & 556 & 32 & 8.00 & -15 & 53.43 & 60.8 & 9.5 & 111 & 0.5 & 125 & 9.0 & 110 & Satl & 0.8 & 1.2 & 11 \\
\hline t. 76 & 744 & 32 & 1.95 & -15 & 34.61 & 77 & 9. & 110 & 0. & 103 & 9. & 110 & Satl & 0.9 & 1.8 & 13 \\
\hline 12 Sept. 76 & 830 & 31 & 59.5 & -15 & 26.4 & 8 & 9.6 & 109 & 0.6 & 103 & 9. & 109 & $\mathrm{C} / \mathrm{C}$ & & & 14 \\
\hline 12 Sept. 76 & 834 & 31 & 59.28 & -15 & 25.71 & 85.8 & 9.2 & 112 & 0.5 & 174 & 9.0 & 109 & Satl & 0.6 & 0.8 & 16 \\
\hline ept. 76 & 930 & 31 & 56.09 & -15 & 16.29 & 94.4 & 9.8 & 111 & 0.8 & 135 & 9.0 & 109 & Satl & 0.5 & 0.9 & 18 \\
\hline 12 Sept. 76 & 1020 & 31 & 53.15 & -15 & 7.35 & 2.6 & 9 & 11 & 0.7 & 173 & 9.0 & 109 & Satl & 0.8 & 0.8 & 20 \\
\hline t. 76 & 1056 & 31 & 50.97 & -15 & 1.28 & 108 & 9. & 10 & 0. & 109 & 9 & 109 & Satl & 0.5 & 0.6 & 22 \\
\hline 12 Sept. 76 & 1110 & 31 & 50.2 & -14 & 58.8 & 110.4 & 9.0 & 10 & 0.5 & 109 & 8 & 109 & $\mathrm{C} / \mathrm{S}$ & & & 23 \\
\hline 12 Sept. 76 & 1116 & 31 & 49.95 & -14 & 57.79 & 111.3 & 9.8 & 108 & 1.3 & 105 & 8.5 & 109 & Satl & 0.2 & 0.3 & 25 \\
\hline t. 76 & 1125 & 31 & 49.5 & -14 & 56.1 & 112.8 & 6.3 & 108 & 1.3 & 105 & 5.0 & 109 & $\mathrm{C} / \mathrm{S}$ & & & 26 \\
\hline 12 Sept. 76 & 1330 & 31 & 45. & -14 & 41 & 126.0 & 10.3 & 10 & 1. & 10 & 9. & 10 & $\mathrm{C} /$ & & & 27 \\
\hline 12 Sept. 76 & 1410 & 31 & 43.21 & -14 & 33.69 & 132.9 & 9.0 & 108 & 0.1 & 351 & 9.0 & 109 & Satl & 3.9 & 2.9 & 29 \\
\hline 12 Sept. 76 & 1556 & 31 & 38.22 & -14 & 16.06 & 148.7 & 9.1 & 109 & 0.1 & 101 & 9.0 & 109 & Satl & 0.2 & 1.8 & 31 \\
\hline 12 Sept. 76 & 1752 & 31 & 32.51 & -13 & 56.48 & 166.3 & 8.5 & 11 & 0.6 & 250 & 9. & 109 & Satl & 0.3 & 1.9 & 33 \\
\hline 12 Sept. 76 & 1914 & 31 & 28.22 & -13 & 43.77 & 178.0 & 9.2 & 107 & 0.4 & 47 & 9.0 & 109 & Satl & 0.9 & 1.4 & 35 \\
\hline t. 76 & 2018 & 31 & 25.3 & -13 & 32.7 & 18 & 9. & 11 & 0 . & 15 & 9. & 10 & $\mathrm{~S}$ & 0.5 & 1.1 & 37 \\
\hline t. 7 & 2122 & 3 & 22.0 & 12 & 22.01 & 19 & 9. & & 0 & 20 & 9. & 109 & $\mathrm{~S}$ & 0 . & 1.1 & 39 \\
\hline 12 Sept. 76 & 222 & 31 & 19.47 & -13 & 15.65 & 203.5 & 9.0 & 113 & 0.7 & 204 & 9.0 & 109 & Satl & 0.7 & 0.7 & 41 \\
\hline 12 Sept. 76 & 2310 & 31 & 15.44 & -13 & 4.74 & 213.7 & 9.1 & 11 & 0.5 & 185 & 9.0 & 109 & Satl & 0.8 & 1.1 & 43 \\
\hline 13 Sept. 76 & 155 & 31 & 6.0 & -12 & 37.5 & 238.8 & 5.1 & 114 & 0.5 & 185 & 5.0 & 109 & $\mathrm{C} / \mathrm{S}$ & & & 44 \\
\hline
\end{tabular}


TABLE 1 - Continued

\begin{tabular}{|c|c|c|c|c|c|c|c|c|c|c|c|c|c|c|c|c|}
\hline \multirow[b]{2}{*}{ Date } & \multirow[b]{2}{*}{ Time } & \multicolumn{2}{|c|}{ Latitude } & \multicolumn{2}{|c|}{ Longitude } & \multirow[b]{2}{*}{ Dist. } & \multicolumn{2}{|c|}{ Actual } & \multicolumn{2}{|c|}{ Drift } & \multicolumn{2}{|c|}{ Dr } & & & Drift & \\
\hline & & Deg. & Min. & Deg. & Min. & & Speed & Cse. & Speed & Hed. & Speed & Cse. & Cmnt. & Dist. & Time & No \\
\hline 13 Sept. 76 & 22 & 31 & 5.80 & -12 & 36.87 & 239.4 & 5.7 & 110 & 0.7 & 114 & 5.0 & 109 & Satl & 1.4 & 2.9 & 46 \\
\hline 13 Sept. 76 & 222 & 31 & 5.2 & -12 & 34.8 & 241.3 & 9.7 & 109 & 0.7 & 114 & 9.0 & 109 & $\mathrm{C} / \mathrm{S}$ & & & 47 \\
\hline 13 Sept. 76 & 230 & 31 & 4.7 & -12 & 33.4 & 242.6 & 9.7 & 101 & 0.7 & 114 & 9.0 & 100 & $\mathrm{C} / \mathrm{C}$ & & & 48 \\
\hline 13 Sept. 76 & 346 & 31 & 2.39 & -12 & 19.30 & 254.9 & 8.2 & 98 & 0.8 & 296 & 9.0 & 100 & Satl & 1.3 & 1.7 & 50 \\
\hline 13 Sept. 76 & 410 & 31 & 1.9 & -12 & 15.5 & 258.2 & 8.2 & 108 & 0.8 & 296 & 9.0 & 109 & $\mathrm{C} / \mathrm{C}$ & & & 51 \\
\hline 13 Sept. 76 & 58 & 30 & 59.44 & -12 & 6.76 & 266.1 & 8.8 & 107 & 0.3 & 344 & 9.0 & 109 & Satl & 1.2 & 1.4 & 53 \\
\hline 13 Sept. 76 & 558 & 30 & 57.3 & -11 & 58.6 & 273.4 & 5.0 & 73 & 0.3 & 344 & 5.0 & 77 & $\mathrm{C} / \mathrm{CS}$ & & & 54 \\
\hline 13 Sept. 76 & 654 & 30 & 58.62 & -11 & 53.38 & 278.1 & 5.5 & 78 & 0.5 & 87 & 5.0 & 77 & Satl & 0.6 & 1.8 & 56 \\
\hline 13 Sept. 76 & 758 & 30 & 59.8 & -11 & 46.7 & 283.9 & 3.0 & 79 & 0.5 & 87 & 2.5 & 77 & $\mathrm{C} / \mathrm{S}$ & & & 57 \\
\hline 13 Sept. 76 & 80 & 30 & 59.9 & -11 & 46.6 & 284.0 & 5.5 & 78 & 0.5 & 87 & 5.0 & 77 & $\mathrm{C} / \mathrm{S}$ & & & 58 \\
\hline 13 Sept. 76 & 928 & 31 & 1.56 & -11 & 37.41 & 292.1 & 5.6 & 77 & 0.6 & 76 & 5.0 & 77 & Satl & 1.3 & 2.6 & 60 \\
\hline 13 Sept. 76 & 948 & 31 & 1.98 & -11 & 35.30 & 293.9 & 6.1 & 76 & 1.1 & 72 & 5.0 & 77 & Satl & 0.2 & 0.3 & 62 \\
\hline 13 Sept. 76 & 1026 & 31 & 2.90 & -11 & 30.96 & 297.7 & 4.7 & 79 & 0.3 & 236 & 5.0 & 77 & Satl & 0.7 & 0.6 & 64 \\
\hline 13 Sept. 76 & 1035 & 31 & 3.0 & -11 & 30.2 & 298.4 & 4.9 & 343 & 0.3 & 236 & 5.0 & 347 & $\mathrm{C} / \mathrm{C}$ & & & 65 \\
\hline 13 Sept. 76 & 1045 & 31 & 3.8 & -11 & 30.4 & 299.3 & 5.3 & 256 & 0.3 & 236 & 5.0 & 257 & $\mathrm{C} / \mathrm{C}$ & & & 66 \\
\hline 13 Sept. 76 & 1232 & 31 & 1.5 & -11 & 41.2 & 308.8 & 4.7 & 79 & 0.3 & 236 & 5.0 & 77 & $\mathrm{C} / \mathrm{C}$ & & & 67 \\
\hline 13 Sept. 76 & 1322 & 31 & 2.2 & -11 & 36.7 & 312.7 & 5.3 & 256 & 0.3 & 236 & 5. & 257 & $\mathrm{C} / \mathrm{C}$ & & & 68 \\
\hline 13 Sept. 76 & 1346 & 31 & 1.72 & -11 & 39.11 & 314.8 & 5.0 & 257 & 0.0 & 0 & 5.0 & 257 & S415 & 1.2 & 3.3 & 70 \\
\hline 13 Sept. 76 & 1346 & 31 & 1.7 & -11 & 39.1 & 314.8 & 0.0 & 0 & 0.0 & 0 & 0.0 & 500 & Stop & & & 71 \\
\hline 28 Sept. 76 & 235 & 31 & 1.72 & -11 & 39.11 & 314.8 & 1.5 & 8 & 1.5 & 8 & 0.0 & 500 & Dr & 0.1 & 369.3 & 73 \\
\hline 28 Sept. 76 & 235 & 31 & 1.7 & -11 & 39.1 & 314.8 & 6.4 & 20 & 1.5 & 8 & 5.0 & 23 & $\mathrm{U} / \mathrm{W}$ & & & 74 \\
\hline pt. 76 & 2320 & 31 & 3.2 & -11 & 38.5 & 316.4 & 5.4 & 19 & 1. & 8 & 4. & 23 & & & & 75 \\
\hline 28 Sept. 76 & 2330 & 31 & 4.1 & -11 & 38.1 & 317.3 & 5.5 & 15 & 1.5 & 8 & 4. & 17 & $\mathrm{C} / \mathrm{C}$ & & & 76 \\
\hline 28 Sept. 76 & 2345 & 31 & 5.4 & -11 & 37.7 & 318.7 & 4.5 & 14 & 1.5 & 8 & 3. & 17 & $\mathrm{C} / \mathrm{S}$ & & & 77 \\
\hline 29 Sept. 76 & 130 & 31 & 12.99 & -11 & 35.51 & 326.5 & 3.9 & 18 & 0.9 & 22 & 3.0 & 17 & Satl & 3.6 & 2.4 & 79 \\
\hline 29 Sept. 76 & 140 & 31 & 13.6 & -11 & 35.3 & 327.1 & 6.9 & 18 & 0.9 & 22 & 6.0 & 17 & $\mathrm{C} / \mathrm{S}$ & & & 80 \\
\hline pt. 76 & 240 & 31 & 20.2 & -11 & 32.8 & 334.0 & 9.9 & 17 & 9 & 22 & 9 & 17 & $\mathrm{C} / \mathrm{S}$ & & & 81 \\
\hline 29 Sept. 76 & 316 & 31 & 25.86 & -11 & 30.72 & 340.0 & 9.2 & 17 & 0. & 26 & 9. & 17 & Satl & 1.7 & 1.8 & 83 \\
\hline 29 Sept. 76 & 438 & 31 & 37.85 & -11 & 26.37 & 352.5 & 8.9 & 16 & 0.2 & 228 & & 17 & $\mathrm{~S}$ & 0.3 & 1.4 & 85 \\
\hline 29 Sept. 76 & 550 & 31 & 48.05 & -11 & 22.83 & 363.2 & 8.6 & 19 & 0.5 & 153 & 9.0 & 17 & Satl & 0.2 & 1.2 & 87 \\
\hline 29 Sept. 76 & 736 & 32 & 2.43 & -11 & 16.86 & 378.4 & 8.6 & 2 & 2.4 & 270 & 9.0 & 17 & Satl & 1.0 & 1.8 & 89 \\
\hline pt. 76 & 84 & 32 & 6.45 & -11 & 16.73 & 382.4 & 9.0 & 20 & 0 & 10 & 9. & 17 & Satl & 1.2 & 0.5 & 91 \\
\hline 29 Sept. 76 & 89 & 32 & 7.2 & -11 & 16.4 & 383.2 & 5.0 & 22 & 0 . & 10 & 5. & 17 & $\mathrm{C} / \mathrm{S}$ & & & 92 \\
\hline 29 Sept. 76 & 818 & 32 & 7.9 & -11 & 16.1 & 383.9 & 9.0 & 20 & 0.4 & 106 & 9. & 17 & $\mathrm{C} / \mathrm{S}$ & & & 93 \\
\hline 29 Sept. 76 & 934 & 32 & 18.61 & -11 & 11.56 & 395.4 & 9.2 & 19 & 0.4 & 79 & 9.0 & 17 & Satl & 0.7 & 1.5 & 95 \\
\hline 29 Sept. 76 & 954 & 32 & 21.50 & -11 & 10.38 & 398.4 & 9.3 & 16 & 0.3 & 334 & 9.0 & 17 & Satl & 0.2 & 0.3 & 97 \\
\hline 29 Sept. 76 & 125 & 32 & 41.0 & -11 & 4 & 5 & 5. & 14 & 0 & 33 & 5 & 17 & $\mathrm{C} / \mathrm{S}$ & & & 98 \\
\hline 29 Sept. 76 & 1225 & 32 & 42.7 & -11 & 3. & 420.4 & 9.3 & 16 & 0 . & 33 & 9. & 17 & $\mathrm{C} / \mathrm{S}$ & & & 99 \\
\hline 29 Sept. 76 & 1244 & 32 & 45.47 & -11 & 2.50 & 423.3 & 8.8 & 17 & 0.2 & 213 & & 17 & Satl & 1.0 & 2.8 & 101 \\
\hline 29 Sept. 76 & 1310 & 32 & 49.1 & -11 & 1.2 & 427.1 & 8.9 & 88 & 0.2 & 213 & 9.0 & 87 & $\mathrm{C} / \mathrm{C}$ & & & 102 \\
\hline 29 Sept. 76 & 1331 & 32 & 49.3 & -10 & 57.5 & 430.2 & 8.9 & 86 & 0.2 & 213 & 9.0 & 85 & $\mathrm{C} / \mathrm{C}$ & & & 103 \\
\hline 29 Sept. 76 & 1350 & 32 & 4 & -10 & 54.1 & 433.1 & 6.7 & 81 & 0 . & 213 & & 80 & S & & & 104 \\
\hline 29 Sept. 76 & 1430 & 32 & 50.16 & -10 & 48.89 & 437.5 & 7.0 & 88 & 1. & 16 & 6 & 80 & Satl & 0.3 & 1.8 & 106 \\
\hline 29 Sept. 76 & 1436 & 32 & 50.18 & -10 & 48 & 438.2 & 6.8 & 80 & 0. & 0 & 6. & 80 & S416 & 0.2 & 0.1 & 108 \\
\hline 29 Sept. 76 & 1436 & 32 & 50.2 & -10 & 48.1 & 438.2 & 0.0 & 0 & 0.0 & 0 & & 500 & Stop & & & 109 \\
\hline 26 Oct. 76 & 2239 & 32 & 50.18 & -10 & 48.06 & 438.2 & 1.3 & 97 & 1.3 & 97 & 0.0 & 500 & Dep & 0.1 & 656.0 & 111 \\
\hline Oct. 76 & 2239 & 32 & 50.2 & -10 & 48.1 & 438.2 & & & & 97 & & 270 & $\mathrm{U} / \mathrm{W}$ & & & 112 \\
\hline 26 Oct. 76 & 2240 & 32 & 50.2 & -10 & 48.2 & 438.3 & 5.5 & & 1. & 9 & & 268 & $\mathrm{C} / \mathrm{C}$ & & & 113 \\
\hline 26 Oct. 76 & 2310 & 32 & 50.0 & -10 & 51.4 & 441.1 & 8.3 & 267 & 1.3 & 97 & 9.6 & 268 & $\mathrm{I} / \mathrm{S}$ & & & 114 \\
\hline 27 Oct. 76 & 00 & 32 & 49.6 & -10 & 59.7 & 448.0 & 8.3 & 267 & 1.3 & 97 & 9.6 & 268 & & & & 115 \\
\hline 27 Oct. 76 & 038 & 32 & 49.24 & -11 & 5.95 & 453.3 & 8.2 & 266 & 1.4 & 99 & 9.6 & 268 & Satl & 2.6 & 2.0 & 117 \\
\hline 27 Oct. 76 & 134 & 32 & & -11 & 15.04 & 460.9 & & & & & & 268 & $\mathrm{Sa}$ & 1. & 0.9 & 119 \\
\hline 27 Oct. 76 & 412 & 32 & 46.52 & -11 & 40. & 482.4 & 8.1 & 26 & 1. & 11 & 9. & 268 & Satl & 4.1 & 2.6 & 121 \\
\hline 27 Oct. 76 & 50 & 32 & 45.6 & -11 & 48. & 488 & 8. & & 1. & & & & $\mathrm{C} / \mathrm{C}$ & & & 122 \\
\hline 27 Oct. 76 & 526 & 32 & 45.23 & -11 & 52.23 & 492.4 & 7.2 & 264 & 2.6 & 10 & 9.6 & 270 & Satl & 2.3 & 1.2 & 124 \\
\hline 27 Oct. 76 & 558 & 32 & 44.80 & -11 & 56.78 & 496.3 & 8.3 & 263 & 1.6 & 126 & 9.6 & 270 & Satl & 1.4 & 0.5 & 126 \\
\hline 27 Oct. 76 & 626 & 32 & & -12 & 1.38 & 500 & 8.6 & & 1. & 131 & 9. & 270 & Satl & 0.8 & 0.5 & 128 \\
\hline 27 Oct. 76 & 650 & 32 & 44.0 & -12 & 5.5 & 503.6 & & & & & & & $\mathrm{C} / \mathrm{C}$ & & & 129 \\
\hline 27 Oct. 76 & 712 & 32 & 43.78 & -12 & 9.18 & 506 & 8.8 & 26 & 1. & 13 & 9. & 272 & Satl & 1.1 & 0.8 & 131 \\
\hline 27 Oct. 76 & 814 & 32 & 43.23 & -12 & 19.93 & 515.8 & 8.7 & 266 & 1.3 & & & 272 & Satl & 1.3 & 1.0 & 133 \\
\hline 27 Oct. 76 & 1148 & 32 & 41.26 & -12 & 56.63 & 546.7 & 7.9 & 266 & 1.9 & 117 & 9.6 & 272 & . & 4.7 & 3.6 & 135 \\
\hline 27 Oct. 76 & 1514 & 32 & 39.54 & -13 & 28.98 & 574.0 & 8.1 & 270 & 1.6 & 102 & 9.6 & 272 & Satl & 6.4 & 3.4 & 137 \\
\hline 27 Oct. 76 & 166 & 32 & 39.54 & -13 & 37.30 & 581.0 & 7.6 & 269 & 2.1 & 101 & 9.6 & 272 & Satl & 1.4 & 0.9 & 139 \\
\hline
\end{tabular}


TABLE 1 - Continued

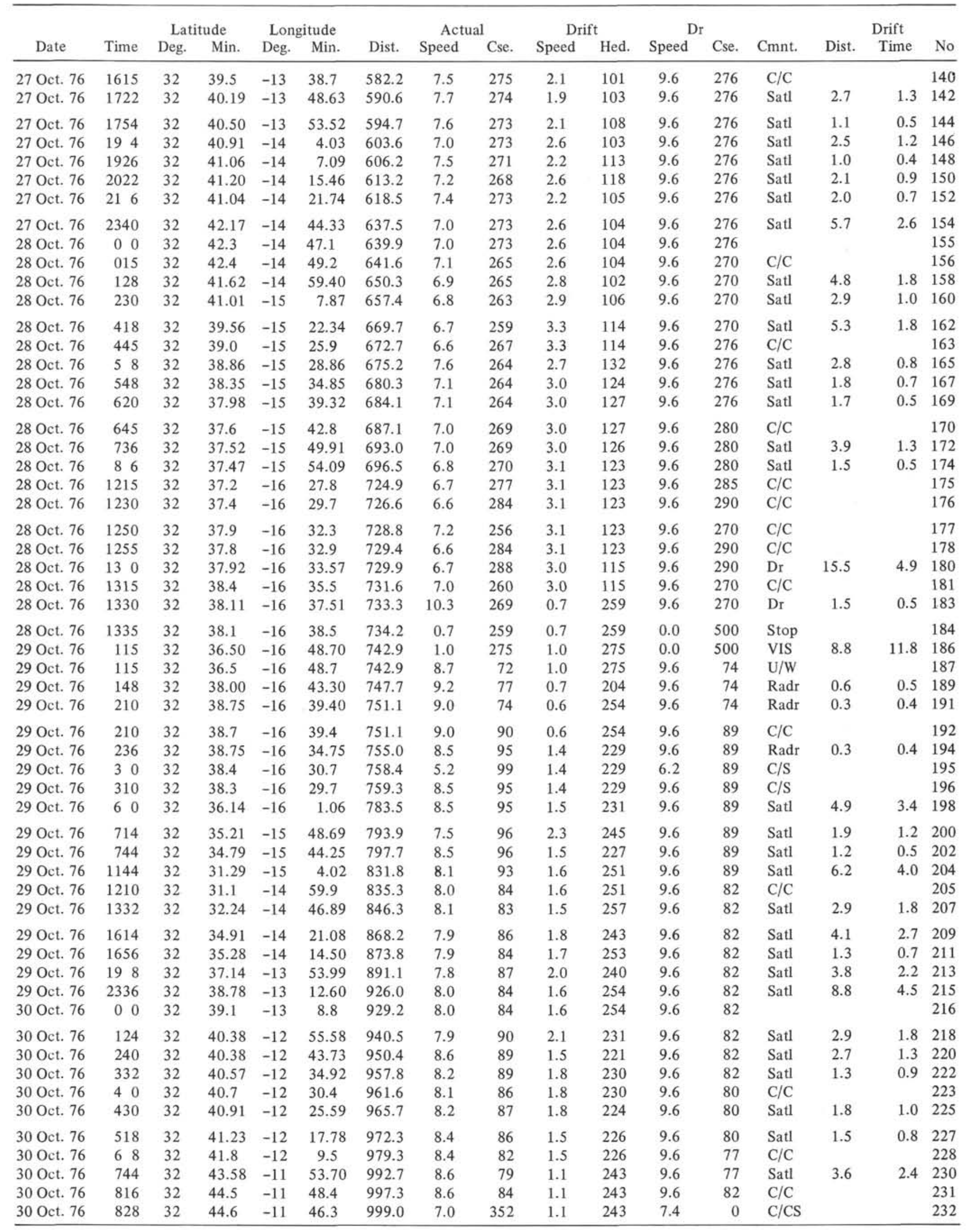


TABLE 1 - Continued

\begin{tabular}{|c|c|c|c|c|c|c|c|c|c|c|c|c|c|c|c|c|}
\hline \multirow[b]{2}{*}{ Date } & \multirow[b]{2}{*}{ Time } & \multicolumn{2}{|c|}{ Latitude } & \multicolumn{2}{|c|}{ Longitude } & \multirow[b]{2}{*}{ Dist. } & \multicolumn{2}{|c|}{ Actual } & \multicolumn{2}{|c|}{ Drift } & \multicolumn{2}{|c|}{$\mathrm{Dr}$} & \multirow[b]{2}{*}{ Cmnt. } & \multicolumn{3}{|c|}{ Drift } \\
\hline & & Deg. & Min. & Deg. & Min. & & Speed & Cse. & Speed & Hed. & Speed & Cse. & & Dist. & Time & No \\
\hline 30 Oct. 76 & 833 & 32 & 45.2 & -11 & 46.4 & 999.6 & 7.2 & 342 & 1.1 & 243 & 7.4 & 350 & $\mathrm{C} / \mathrm{C}$ & & & 233 \\
\hline 30 Oct. 76 & 838 & 32 & 45.8 & -11 & 46.7 & 1000.2 & 8.6 & 84 & 1.1 & 243 & 9.6 & 82 & $\mathrm{C} / \mathrm{CS}$ & & & 234 \\
\hline 30 Oct. 76 & 856 & 32 & 46.0 & -11 & 43.6 & 1002.7 & 6.4 & 85 & 1.1 & 243 & 7.4 & 82 & $\mathrm{C} / \mathrm{S}$ & & & 235 \\
\hline 30 Oct. 76 & 92 & 32 & 46.1 & -11 & 42.8 & 1003.4 & 8.0 & 187 & 1.1 & 243 & 7.4 & 180 & $\mathrm{C} / \mathrm{C}$ & & & 236 \\
\hline 30 Oct. 76 & 915 & 32 & 44.4 & -11 & 43.1 & 1005.1 & 8.6 & 84 & 1.1 & 243 & 9.6 & 82 & $\mathrm{C} / \mathrm{CS}$ & & & 237 \\
\hline 30 Oct. 76 & 1050 & 32 & 45.71 & -11 & 26.98 & 1018.7 & 8.5 & 83 & 1.1 & 251 & 9.6 & 82 & Satl & 3.4 & 3.1 & 239 \\
\hline 30 Oct. 76 & 1236 & 32 & 47.44 & -11 & 9.18 & 1033.8 & 8.6 & 83 & 1.0 & 251 & 9.6 & 82 & Satl & 2.0 & 1.8 & 241 \\
\hline 30 Oct. 76 & 130 & 32 & 47.8 & -11 & 5.1 & 1037.2 & 8.6 & 76 & 1.0 & 251 & 9.6 & 75 & $\mathrm{C} / \mathrm{C}$ & & & 242 \\
\hline 30 Oct. 76 & 140 & 32 & 50.0 & -10 & 55.2 & 1045.8 & 5.2 & 88 & 1.0 & 251 & 6.2 & 85 & $\mathrm{C} / \mathrm{CS}$ & & & 243 \\
\hline 30 Oct. 76 & 1415 & 32 & 50.0 & -10 & 53.7 & 1047.1 & 5.2 & 84 & 1.0 & 251 & 6.2 & 82 & $\mathrm{C} / \mathrm{C}$ & & & 244 \\
\hline 30 Oct. 76 & 1420 & 32 & 50.1 & -10 & 53.2 & 1047.5 & 6.4 & 84 & 1.0 & 251 & 7.4 & 82 & $\mathrm{C} / \mathrm{S}$ & & & 245 \\
\hline 30 Oct. 76 & 1434 & 32 & 50.23 & -10 & 51.42 & 1049.0 & 6.6 & 84 & 0.9 & 244 & 7.4 & 82 & Satl & 2.1 & 2.0 & 247 \\
\hline 30 Oct. 76 & 1452 & 32 & 50.4 & -10 & 49.1 & 1051.0 & 6.6 & 93 & 0.9 & 244 & 7.4 & 90 & $\mathrm{C} / \mathrm{C}$ & & & 248 \\
\hline 30 Oct. 76 & 1453 & 32 & 50.4 & -10 & 49.0 & 1051.1 & 6.7 & 99 & 0.9 & 244 & 7.4 & 95 & $\mathrm{C} / \mathrm{C}$ & & & 249 \\
\hline 30 Oct. 76 & 1455 & 32 & 50.4 & -10 & 48.7 & 1051.3 & 6.7 & 104 & 0.9 & 244 & 7.4 & 100 & $\mathrm{C} / \mathrm{C}$ & & & 250 \\
\hline 30 Oct. 76 & 1457 & 32 & 50.3 & -10 & 48.4 & 1051.5 & 6.8 & 115 & 0.9 & 244 & 7.4 & 110 & $\mathrm{C} / \mathrm{C}$ & & & 251 \\
\hline 30 Oct. 76 & 150 & 32 & 50.18 & -10 & 48.06 & 1051.9 & 7.4 & 110 & 0. & 0 & 7.4 & 110 & S416 & 0.4 & 0.4 & 253 \\
\hline 30 Oct. 76 & 150 & 32 & 50.2 & -10 & 48.1 & 1051.9 & 0.0 & 0 & 0.0 & 0 & 0.0 & 500 & Stop & & & 254 \\
\hline 8 Nov. 76 & 1531 & 32 & 50.18 & -10 & 48.06 & 1051.9 & 0.5 & 77 & 0.5 & 77 & 0.0 & 500 & Dep & 0.1 & 216.5 & 256 \\
\hline 8 Nov. 76 & 1531 & 32 & 50.2 & -10 & 48.1 & 1051.9 & 7.0 & 292 & 0.5 & 77 & 7.4 & 290 & $\mathrm{U} / \mathrm{W}$ & & & 257 \\
\hline 8 Nov. 76 & 1614 & 32 & 52.08 & -10 & 53.54 & 1056.9 & 6.9 & 290 & 0.5 & 116 & 7.4 & 290 & Satl & 0.4 & 0.7 & 259 \\
\hline 8 Nov. 76 & 1634 & 32 & 52.85 & -10 & 56.12 & 1059.2 & 7.7 & 287 & 0. & 235 & 7.4 & 290 & Satl & 0.2 & 0.3 & 261 \\
\hline 8 Nov. 76 & 1656 & 32 & 53.66 & -10 & 59.35 & 1062.0 & 6.3 & 288 & 1.1 & 119 & 7.4 & 290 & Satl & 0.3 & 0.4 & 263 \\
\hline 8 Nov. 76 & 171 & 32 & 53.8 & -11 & 0.0 & 1062.5 & 8.5 & 289 & 1.1 & 119 & 9.6 & 290 & $\mathrm{C} / \mathrm{S}$ & & & 264 \\
\hline 8 Nov. 76 & 1814 & 32 & 57.19 & -11 & 11.66 & 1072.9 & 8.5 & 292 & 1.2 & 93 & 9.6 & 290 & Satl & 1.4 & 1.3 & 266 \\
\hline 8 Nov. 76 & 1850 & 32 & 59.1 & -11 & 17.3 & 1078.0 & 8.4 & 265 & 1.2 & 93 & 9.6 & 266 & $\mathrm{C} / \mathrm{C}$ & & & 267 \\
\hline 8 Nov. 76 & 2316 & 32 & 55.86 & -12 & 1.51 & 1115.3 & 8.0 & 268 & 1.6 & 78 & 9.6 & 266 & Satl & 6.1 & 5.0 & 269 \\
\hline 9 Nov. 76 & 00 & 32 & 55.6 & -12 & 8.5 & 1121.2 & 8.0 & 268 & 1.6 & 78 & 9.6 & 266 & & & & 270 \\
\hline 9 Nov. 76 & 030 & 32 & 55.5 & -12 & 13.3 & 1125.2 & 8.1 & 270 & 16 & 78 & 9.6 & 268 & $\mathrm{C} / \mathrm{C}$ & & & 271 \\
\hline 9 Nov. 76 & 142 & 32 & 55.46 & -12 & 24.82 & 1134.8 & 8.7 & 271 & 1.0 & 64 & 9.6 & 268 & Satl & 3.9 & 2.4 & 273 \\
\hline 9 Nov. 76 & 152 & 32 & 55.5 & -12 & 26.6 & 1136.3 & 8.1 & 271 & 1.0 & 64 & 9.0 & 268 & $\mathrm{C} / \mathrm{S}$ & & & 274 \\
\hline 9 Nov. 76 & 328 & 32 & 55.65 & -12 & 42.00 & 1149.3 & 7.7 & 264 & 1.4 & 109 & 9.0 & 268 & Satl & 1.8 & 1.8 & 276 \\
\hline 9 Nov. 76 & 345 & 32 & 55.4 & -12 & 44.6 & 1151.5 & 7.7 & 262 & 1.4 & 109 & 9.0 & 266 & $\mathrm{C} / \mathrm{C}$ & & & 277 \\
\hline 9 Nov. 76 & 76 & 32 & 51.8 & -13 & 15.2 & 1177.4 & 8.3 & 262 & 1.4 & 109 & 9.6 & 266 & $\mathrm{C} / \mathrm{S}$ & & & 278 \\
\hline 9 Nov. 76 & 748 & 32 & 51.02 & -13 & 22.12 & 1183.3 & 7.7 & 261 & 2.1 & 105 & 9.6 & 266 & Satl & 6.0 & 4.3 & 280 \\
\hline 9 Nov. 76 & 815 & 32 & 50.5 & -13 & 26.2 & 1186.7 & 7.6 & 269 & 2.1 & 105 & 9.6 & 272 & $\mathrm{C} / \mathrm{C}$ & & & 281 \\
\hline 9 Nov. 76 & 1030 & 32 & 50.04 & -13 & 46.49 & 1203.8 & 8.3 & 268 & 1.4 & 116 & 9.6 & 272 & Satl & 5.7 & 2.7 & 283 \\
\hline 9 Nov. 76 & 1053 & 32 & 49.9 & -13 & 50.3 & 1207.0 & 8.3 & 272 & 1.4 & 116 & 9.6 & 276 & $\mathrm{C} / \mathrm{C}$ & & & 284 \\
\hline 9 Nov. 76 & 1216 & 32 & 50.42 & -14 & 3.86 & 1218.4 & 8.2 & 272 & 1.5 & 119 & 9.6 & 276 & Satl & 2.6 & 1.8 & 286 \\
\hline 9 Nov. 76 & 1336 & 32 & 50.76 & -14 & 16.89 & 1229.4 & 7.8 & 273 & 1.9 & 109 & 9.6 & 276 & Satl & 2.1 & 1.3 & 288 \\
\hline 9 Nov. 76 & 1422 & 32 & 51.06 & -14 & 23.98 & 1235.3 & 7.7 & 273 & 2.0 & 108 & 96 & 276 & Satl & 1.5 & 0.8 & 290 \\
\hline 9 Nov. 76 & 1522 & 32 & 51.46 & -14 & 33.10 & 1243.0 & 8.3 & 274 & 1.4 & 110 & 9.6 & 276 & Satl & 2.0 & 1.0 & 292 \\
\hline 9 Nov. 76 & 1648 & 32 & 52.23 & -14 & 47.20 & 1254.9 & 7.7 & 277 & 1.9 & 94 & 9.6 & 276 & Satl & 2.0 & 1.4 & 294 \\
\hline 9 Nov. 76 & 175 & 32 & 52.5 & -14 & 49.8 & 1257.1 & 7.8 & 262 & 1.9 & 94 & 9.6 & 264 & $\mathrm{C} / \mathrm{C}$ & & & 295 \\
\hline 9 Nov. 76 & 1712 & 32 & 52.35 & -14 & 50.86 & 1258.0 & 8.3 & 261 & 1.4 & 104 & 9.6 & 264 & Satl & 0.8 & 0.4 & 297 \\
\hline 9 Nov. 7 & 858 & 3 & 49.95 & -1 & 2 & 1272.6 & 8.5 & 2 & 1 & 1 & 9.6 & 2 & S. & 2.6 & 1.8 & 299 \\
\hline 9 Nov. 76 & 2224 & 32 & 44.15 & -15 & 41.89 & 1301.6 & 8.7 & 259 & 1.2 & 122 & 9.6 & 264 & Satl & 5.0 & 3.4 & 301 \\
\hline 10 Nov. 76 & 00 & 32 & 41.5 & -15 & 58.1 & 1315.5 & 8.7 & 259 & 1.2 & 122 & 9.6 & 264 & & & & 302 \\
\hline 10 Nov. 76 & 08 & 32 & 41.32 & -15 & 59.47 & 1316.7 & 8.9 & 260 & 1.0 & 127 & 9.6 & 264 & Satl & 2.1 & 1.7 & 304 \\
\hline 10 Nov. 76 & 130 & 32 & 39.1 & -16 & 13.7 & 1328.9 & 8.8 & 264 & 1.0 & 127 & 9.6 & 268 & $\mathrm{C} / \mathrm{C}$ & & & 305 \\
\hline 10 Nov. 76 & 212 & 32 & 38.50 & -16 & 21.00 & 1335.0 & 10.5 & 26 & 1 & 214 & 9. & 268 & Radr & 2.1 & 2.1 & 307 \\
\hline 10 Nov. 76 & 217 & 32 & 38.4 & -16 & 22.0 & 1335.9 & 7.1 & 259 & 1.4 & 214 & 6.2 & 268 & $\mathrm{C} / \mathrm{S}$ & & & 308 \\
\hline 10 Nov. 76 & 220 & 32 & 38.34 & -16 & 22.49 & 1336.3 & 7.1 & 259 & 0.0 & 0 & 6.2 & 268 & $\mathrm{Dr}$ & 0.2 & 0.1 & 309 \\
\hline
\end{tabular}




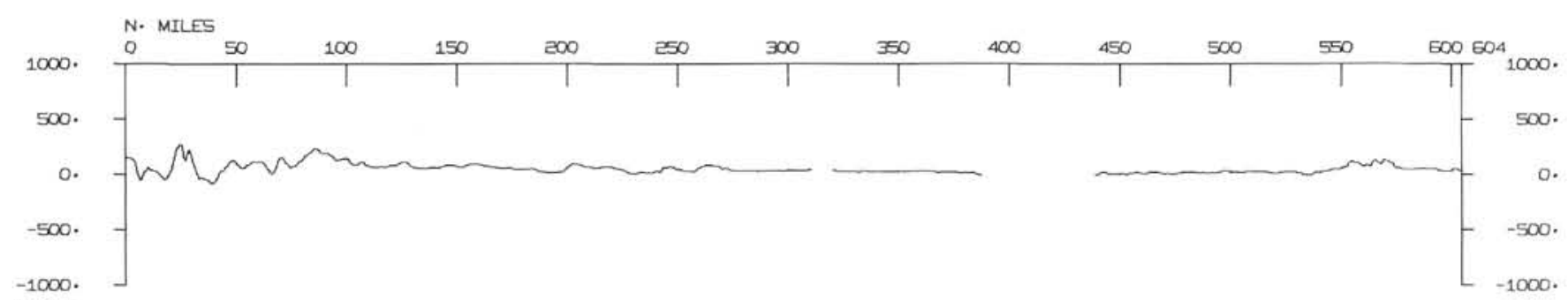

GAMMUS

N. MTIES

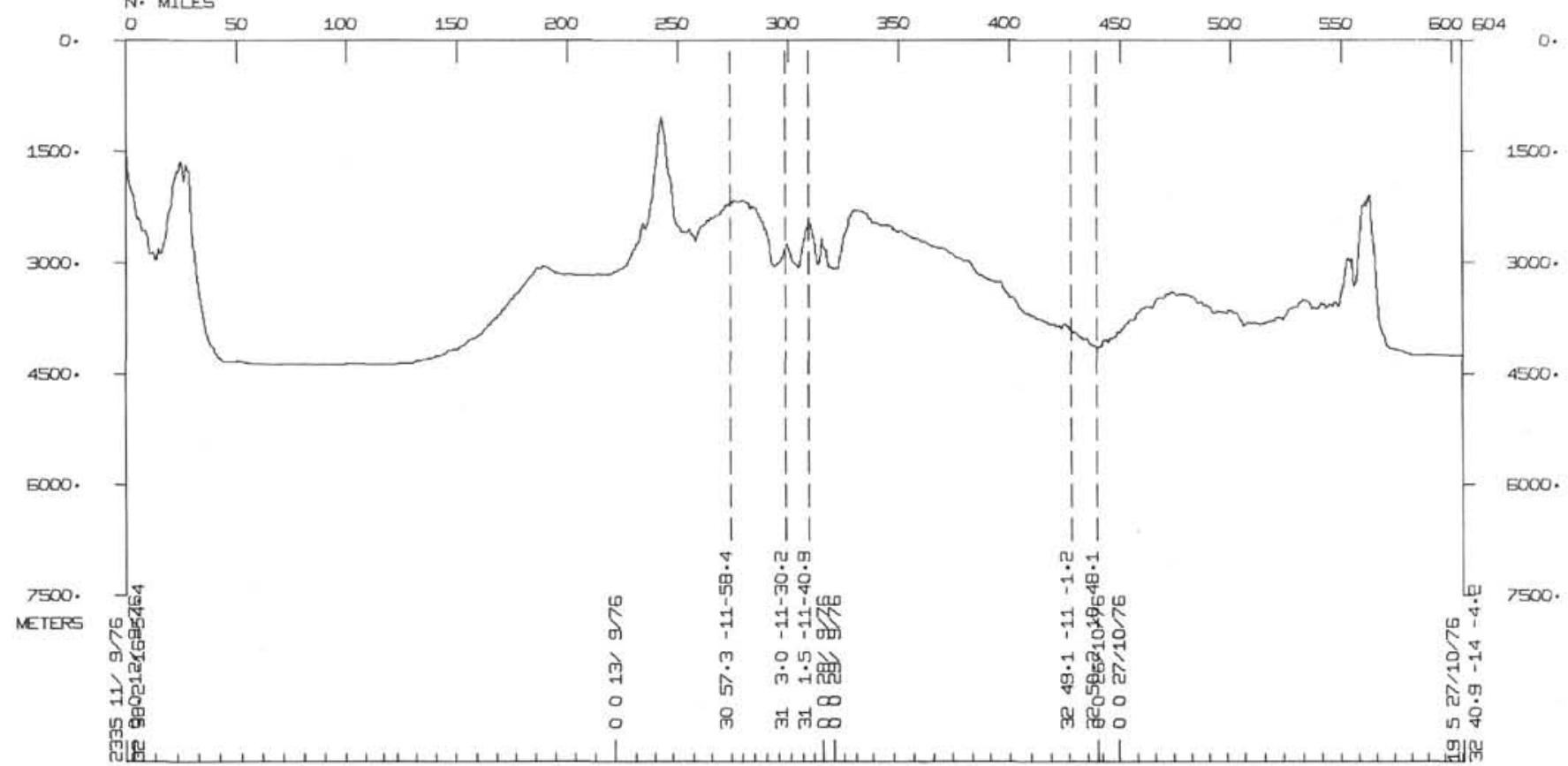

Figure 2. Magnetic and bathymetric profiles along track of Glomar Challenger during Leg 50. 


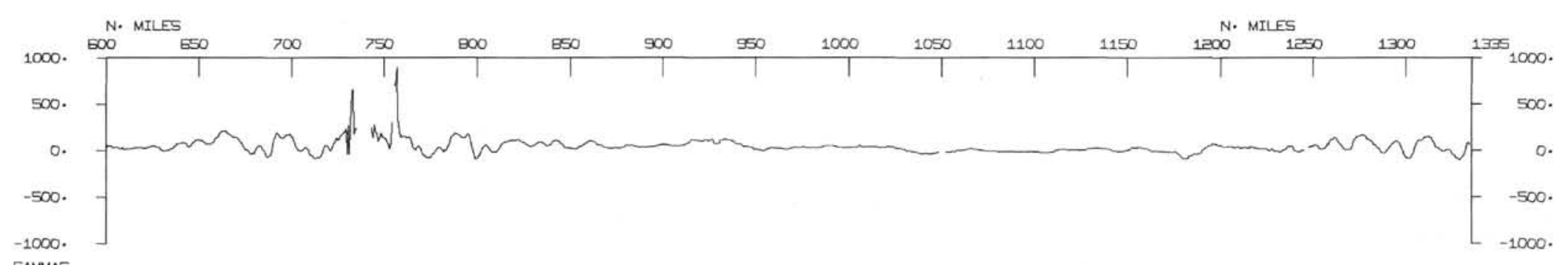

SAMMAS

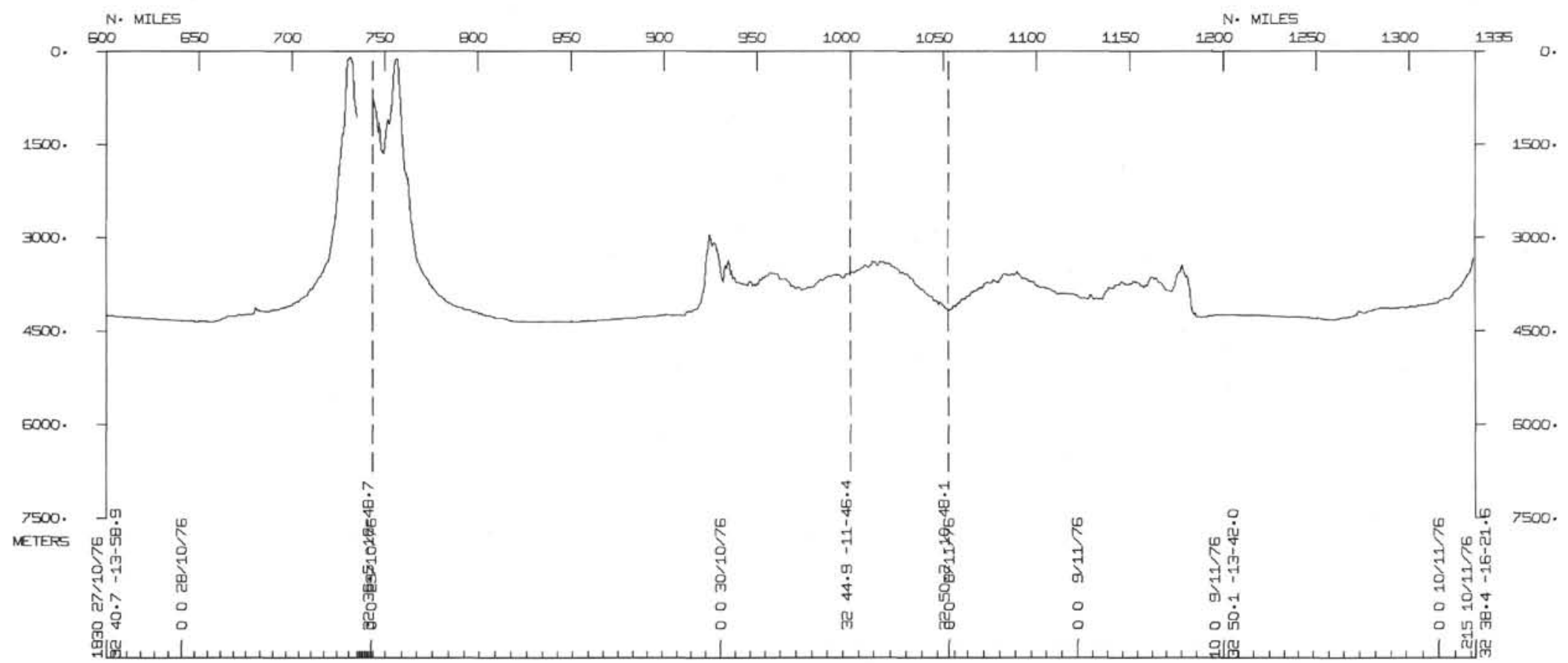

Figure 2. (Continued). 
SHOT POINTS

‥ \& \& \& \& \& \& \& \& \& \& \& \& \& \& \&

\section{SITES $370 / 416$}

(PROJECTED)

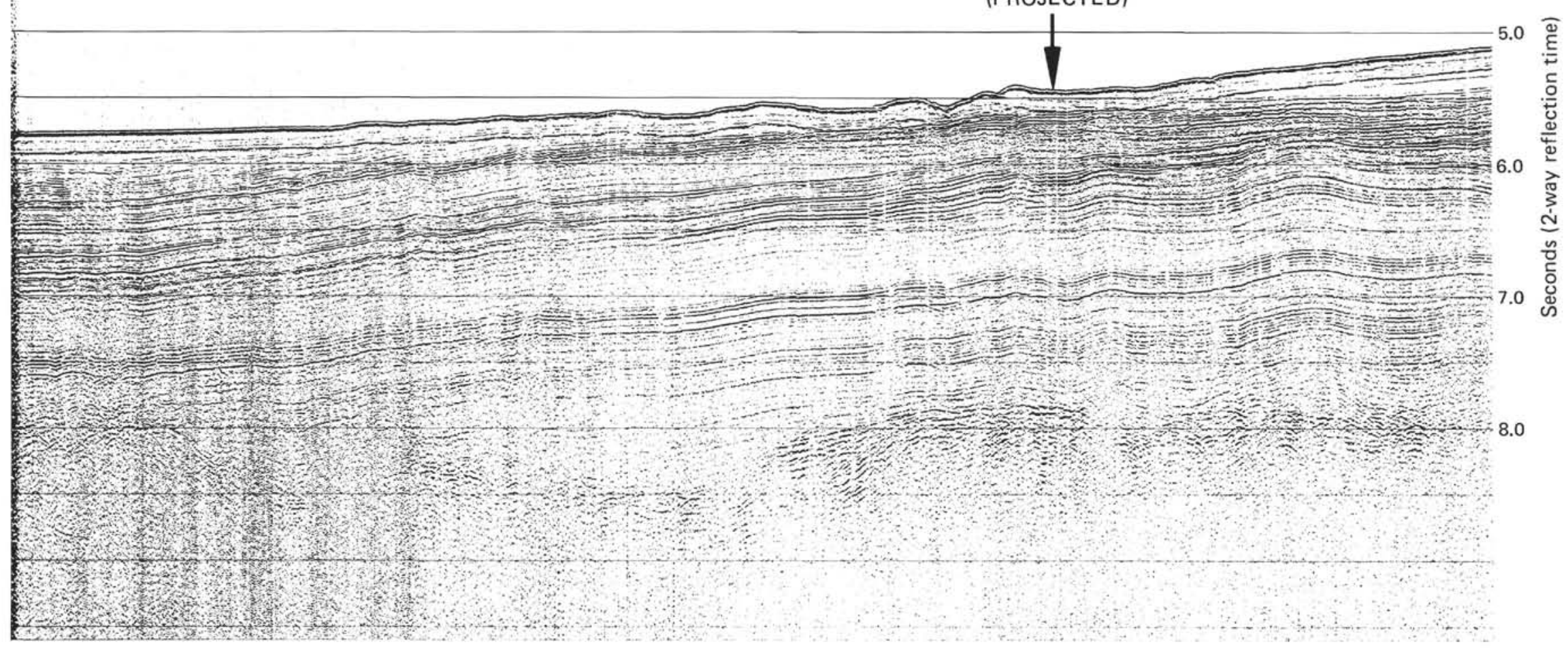

Figure 4. Multifold seismic profile made from R/V Meteor during cruise 3902. 


\section{SHOT POINTS}

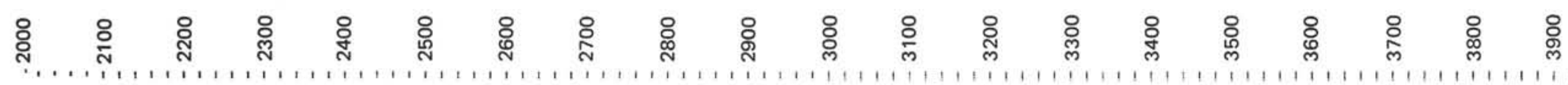

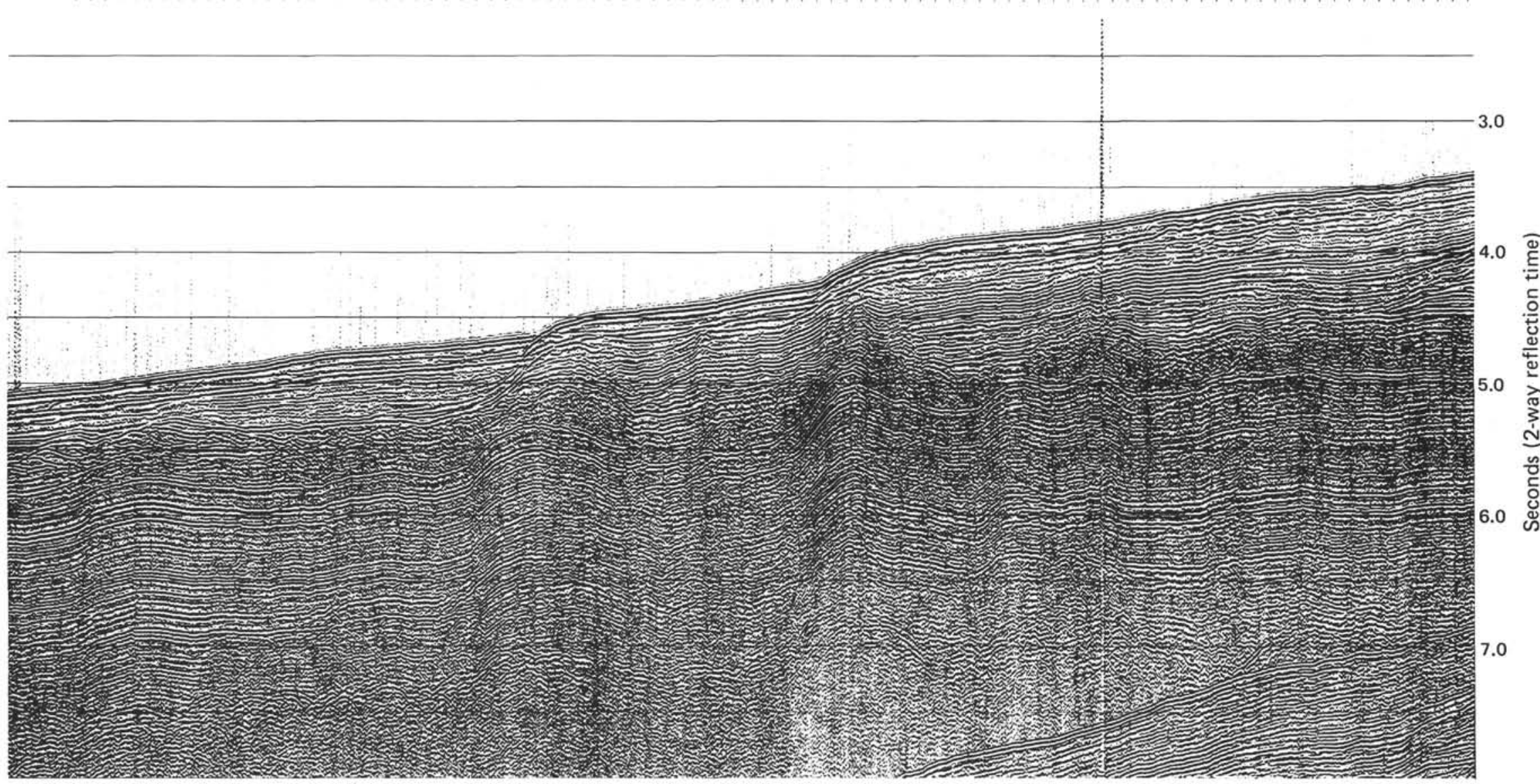

Figure 4. (Continued). 
SHOT POINTS

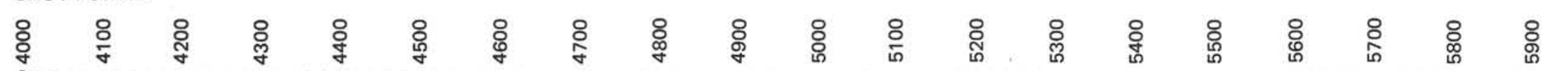

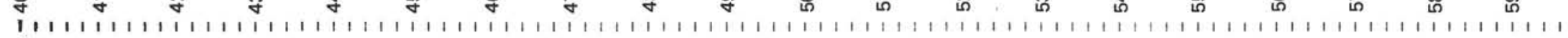

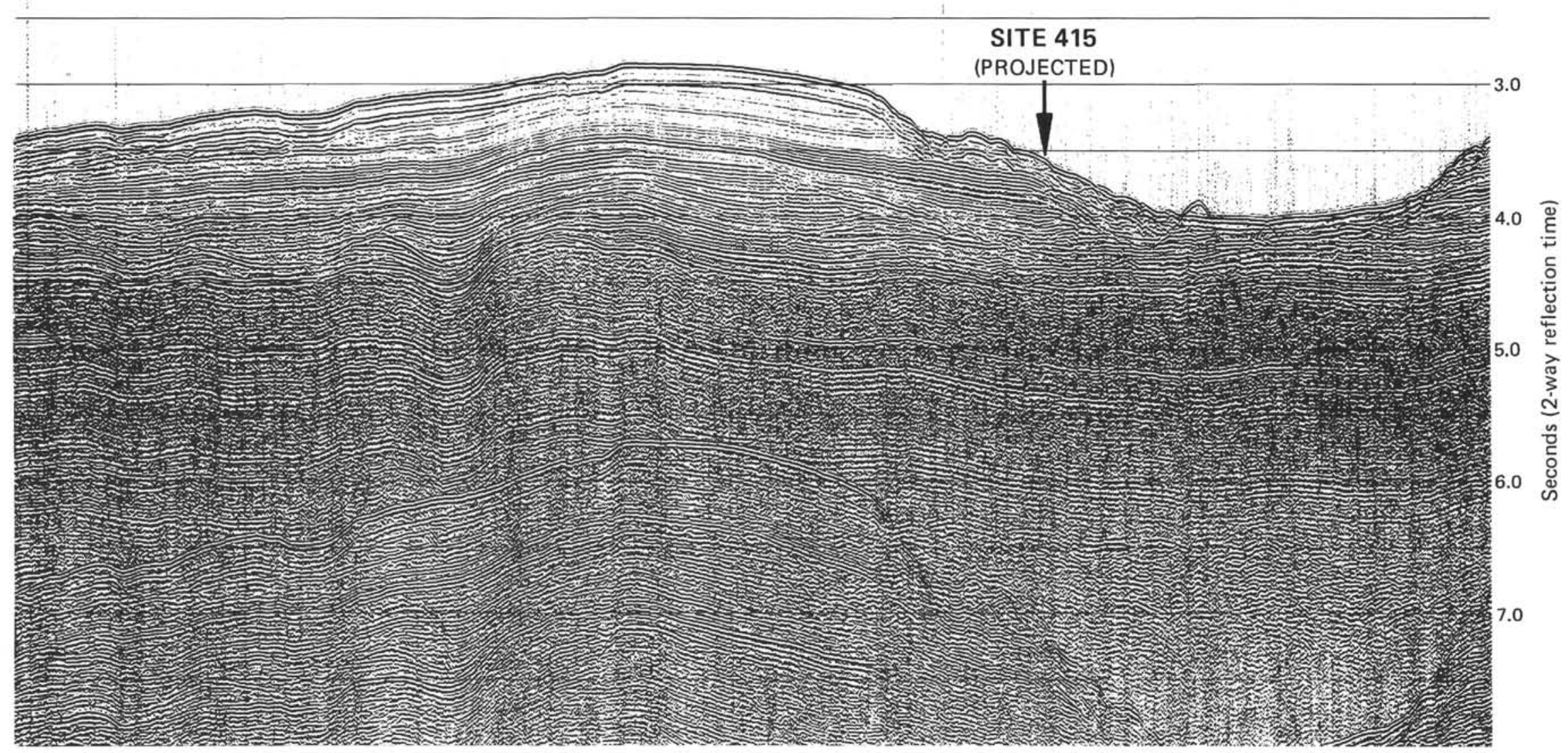

Figure 4. (Continued). 


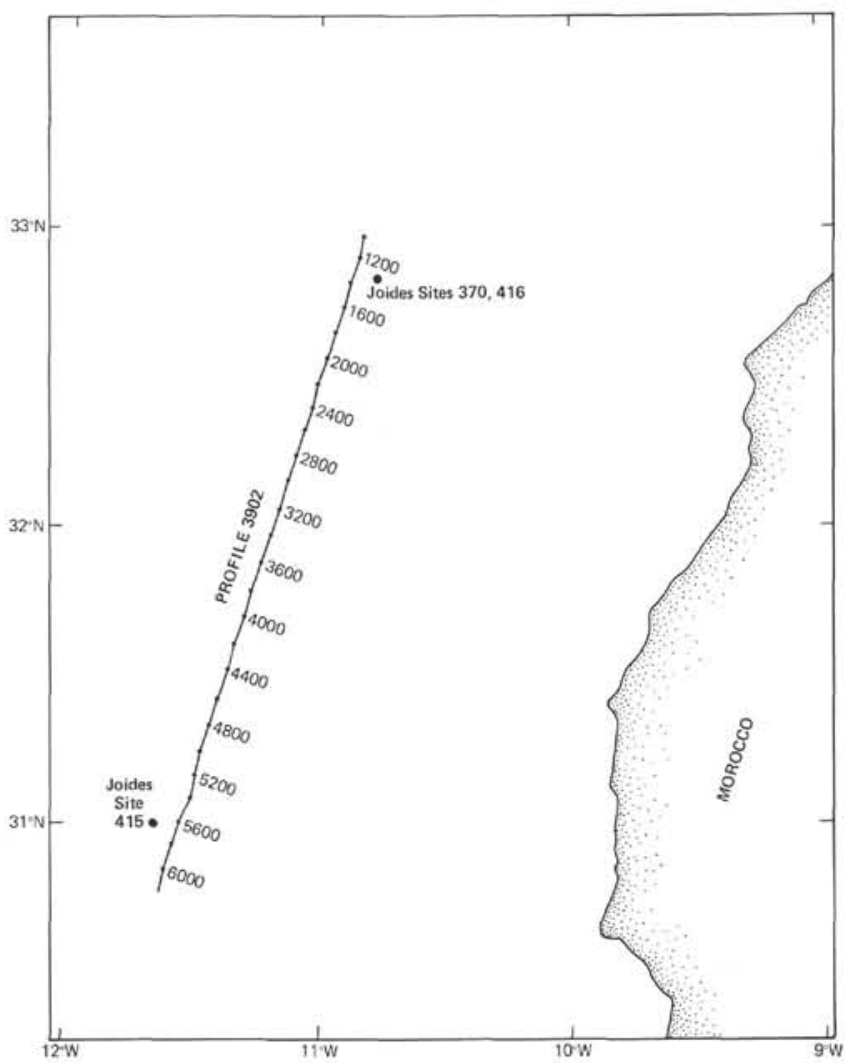

Figure 5. Portion of track of $\mathrm{R} / \mathrm{V}$ Meteor on cruise 3902, showing line covered by Figure 4. 